\title{
Design of A SAFE AND SMART Medicine BoX
}

\author{
Roy ABI ZEID DAOU ${ }^{1,2}$, Khalil KARAM ${ }^{1}$, Hiba ZEIDAN ${ }^{1}$, Ali HAYEK ${ }^{3}$ and \\ Josef BORCSOK ${ }^{3}$
}

\author{
${ }^{1}$ Lebanese German University, Faculty of Public Health, Biomedical \\ Technologies Department, Jounieh, Lebanon \\ r.abizeiddaou@1gu.edu.lb \\ ${ }^{2}$ MART Learning, Education and Research Center, Chananir, Lebanon \\ roydaou@mart-ler.org \\ ${ }^{3}$ Kassel University, Department of Computer Architecture and System \\ Programming, Kassel, Germany \\ Ali.hayek@,uni-kassel.de
}

\begin{abstract}
This paperpresents an advanced medicine intake monitoring and control system. The proposed system consists on a smart medicine box that is designed to help patients taking their medications on time. Two main features are implemented during the design phase of this system: the first one is the safety feature that will alarm the patient in case of not taking and/or bad dosage taking for medication, will keep the medication out of reach of the children and will be used in case of failure of one of the system's components whereas the second feature is the low cost of the system. This box will be linked to a phone application that can signal several alarms mainly when the medications are about to finish, the patient forgets to take his medication or he (she) doesn't take the required dose (or the required number of pills)... This system was tested over 30 different medications and when using ten different mobile phones by more than 25 patients and the results didn't show any faulty alarms.
\end{abstract}

\section{KEYWORDS:}

Medicine box; Safety system; Phone application; Autonomous patient; Smart systems;

\section{INTRODUCTION}

Nowadays, the world and the scientists are targeting more and more the autonomy of the patients by developing advanced tools that can help them being not only autonomous but monitored at the same time. Hence, the proposed system aims to offer one new tools for this community. This system consists on a smart medical box that can alert the patient, via a phone application, about the upcoming schedule to take his medication, the number of remaining pills in the box, an error in the number of pills taken and much more.

So far, lots of systems were developed in order to help elder people and patients being autonomous. From among these systems, we list the one click tool that can control all the room environment of a paralyzed patient [1], the design of a smart home for elder people based on sensors[2][3][4], the development of tools to monitor the fall of patient with movements difficulties[5][6][7], the huge advances in telemedicine systems [8][9],...

Added to that, the use of phone applicationsis getting more familiar in biomedical engineering applications mainly for monitoring purposes. In fact, the availability of such devices with almost DOI: $10.5121 /$ ijbes.2018.5401 
all people has increased its use in several engineering domain, mainly the biomedical industry. However, a special attention should target safety issues especially when sending/receiving data and security issues to deny the availability of data for non-authorized persons.

Concerning the safety issue, although the first norms and regulations related to the medical field were established in 1977 by the International Electro-technical Commission (IEC) [10], its application in the small devices remains limited. However, we can still find few applications where safety is applied as in telemetry [11] and in bioinstrumentation [12].

As for the medical boxes, they were already introduced in several previous works [13][14][15]. However, none of the proposed systems has taken the safety issue into consideration. In fact, no proposed system has shown the software/hardware solution to take if one or more of the components went down.

So, the proposed system consists on a low cost smart medical box, linked to a phone application, helping the patient taking his medications correctly and on time. The system also contains some duplications (at the hardware and software levels) to reinforce the safety and availability issues and to decrease the probability of system failure or faulty alarms.

Although the proposed system can handle one medication type only, it can be used for lots of patients, mainly psychiatric, cancer and Alzheimer patients. In fact, the first category risks not to take medication or to take a bigger quantity than specified by the medical doctor in order to put an end to its life, whereas the second category risks to forget or take medication more than once because of memory problems.

This paper will be divided as follow: in section 2, a general overview of the proposed system, along with its block diagrams, will be presented.Section 3 will show the safety aspect applied to this system with the new block diagram. Section 4 will present the hardware design of this system whereas the software developed for the microcontroller and the phone application will be mentioned in section 5. The next section will show the results of the implementation and the testing of the new tool. At the end, section 7 will present a conclusion and some future works that may enrich this system.

\section{GeNERAL OVERVIEW}

This section will present a general overview of the basic smart medicine box along with its block diagram and the flowchart to show the whole system procedure.

Concerning the system, it is based on a sensor to measure the weight of the pills inside the medicine box. The phone application allows the user to define the weight of a pill (it can be also calculated by the weight sensor within the system) as well as the times to take the medication and the number of pills to be taken each time. The processor within the medicine box will continuously check the availability of the pills as well as the number of pills taken. The connection between the processor and the phone is wireless.

Figure 1 shows the basic block diagram of the system where the interconnection of the processor with the sensor, the alarm system and the phone application (monitoring system) are shown. 


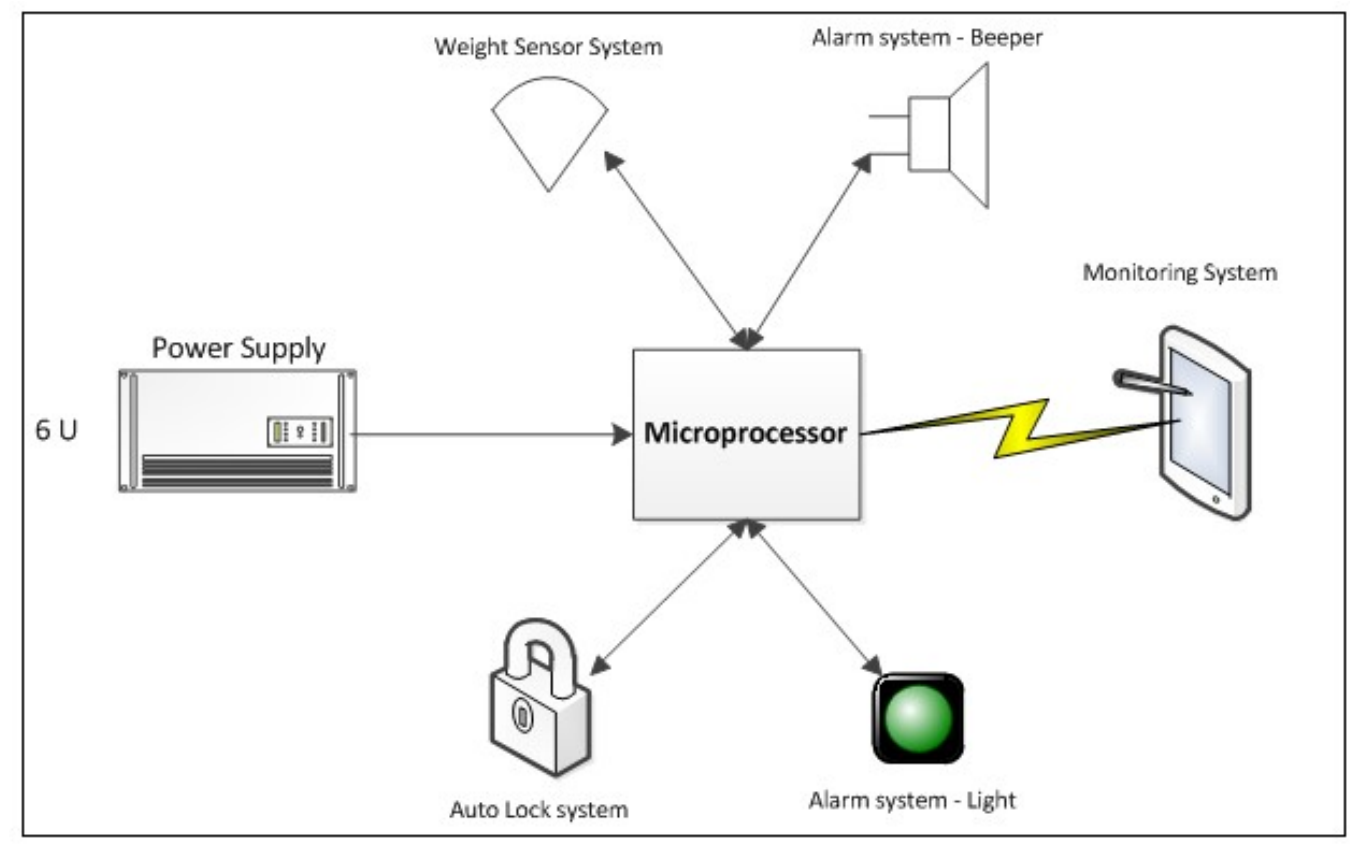

Fig.1 - Block diagram of the system

Figure 2 shows the flowchart of the system. In fact, the data flow, processing and analysis is done using the following scheme: the first step requires from the user to initialize, via the phone application (the monitoring system in general), the main values as the weight of the pill, the timing to take it, the number of pills and their frequency (every day, every week or every month). The data is then sent to the processor in order to synchronize the timing between both devices. Added to that, all alarms are reset from the microprocessor and the box is locked.

The processor tests the timing: whenever 15 minutes are set before the schedule of the medication, the medicine box is unlocked and the processor measures the weight of the pills.

If the door of the box is opened within the next 15 minutes, the calculation of the new pills weight is done and the number of taken pills is defined. If the number of pills is equal to the value set by the patient using the mobile application, the system enters in the sleep mode waiting for the next schedule. However, if the two values do not match, an alarm is set and the processor enters, in the next 15 minutes, a procedure testing continuously the weight of the pills in the box; if the patient takes the required pills, the system enters the sleep mode. Otherwise, an alarm is set to one of his parents (the one who has installed the application) as well as a beeper and a light, placed in the medicine box, are turned on for some moment.

If the door of the box is not opened, an alarm is sent to one of the patient's parents, the beeper and the light are turned on, and the door remains opened upon a request sent by the patient through its phone application. 


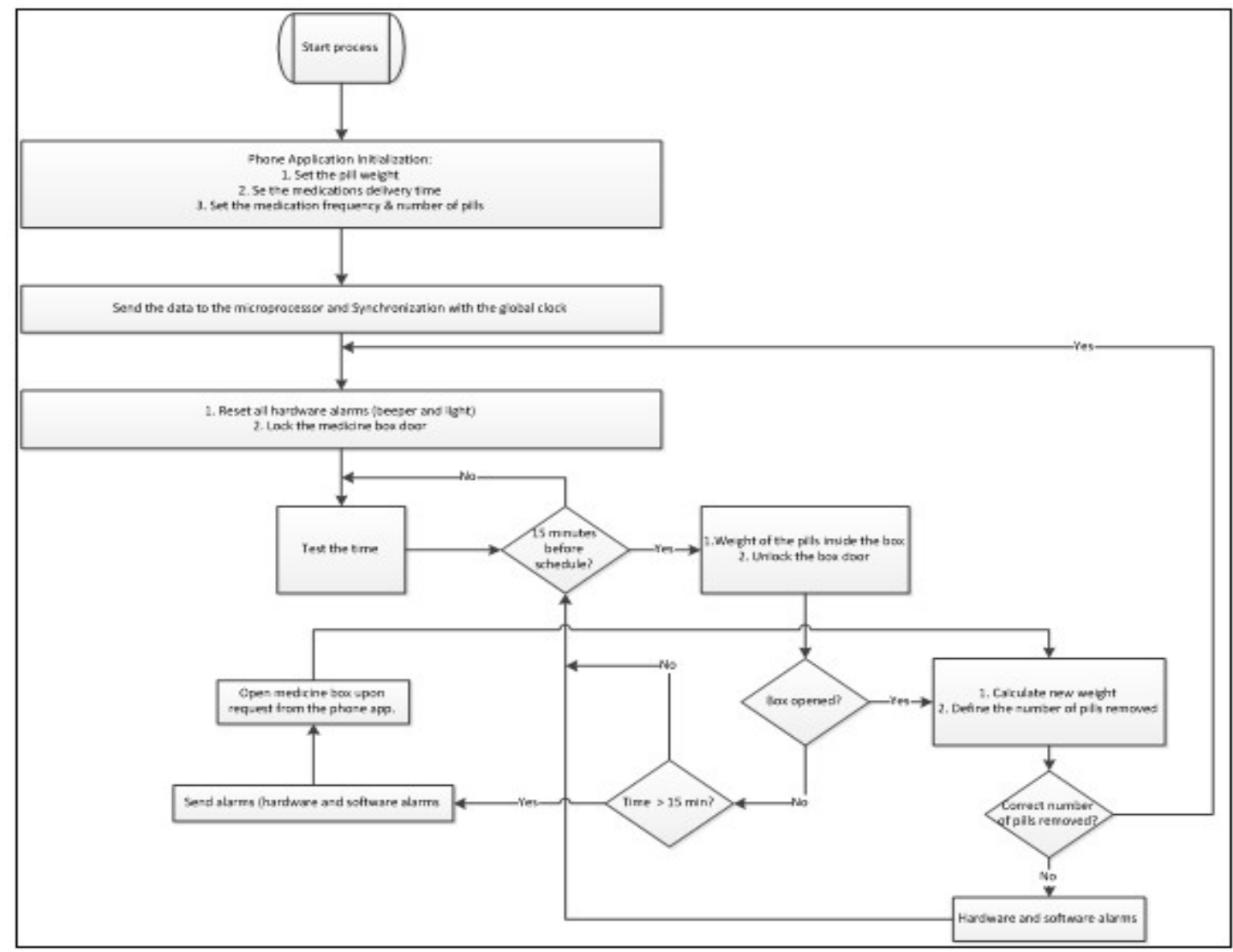

Fig. 2 Flowchart of the system

\section{SAFETY Measurements}

This section will present the security and safety measurements taken into consideration to make sure that the system performs well and the errors caused due to hardware failure, transmission problems,...can be handled automatically. So, we will start first by defining the safety. Then, a new block diagram will be shown pointing at the measurements taken to get a safer system.As for the calculations some characteristics such as MTBF (Mean Time Between Failure) and PFD (Probability of Failure on Demand), detailed information about failure rates of the used components is required. Therefore, the calculation will be part of a future work.

\subsection{SAFETY DEFINITION}

A safe system depends on the probability of failure of the system, the consequences of a failure and the risk of occurrence of a dangerous state. The failure could cause injuries or death of human beings, environmental losses and/or economic losses. The main causes of failure may be hardware (electronic, electrical, mechanical,...), software or communication/transmission failures. 
For this system, several failures can occur. However, the most common ones are sensors, actuators and microprocessor failures as well as communication failures between processor and monitoring unit and problems at power supply source level.

The risks of this system are to take the right dose of medication but the alarm system indicates an error in the number of taken pills or the system would not detect an error or a missing medication intake and will not turn on the alarm system. The safe state can be expressed by sending a message to the patient and his parents via the phone application mentioning that the whole system is no more functional and the patient must check manually for the medication and bring the system into de-energized state. Added to that, the box will remain unlocked.

\subsection{NeW BLOCK DiagraM}

Based on the safety-related aspects argued in the previous paragraph,this section presents the concept for a safety-related system architecture. Thus, figures 3 and 4 shows the new block diagram of the system where the safety measurements are implemented. In fact, figure 3 shows an advanced representation of the block diagram of figure 1 where safetyrelated components are provided whereas figure 4 will show the interconnections of all the components together.

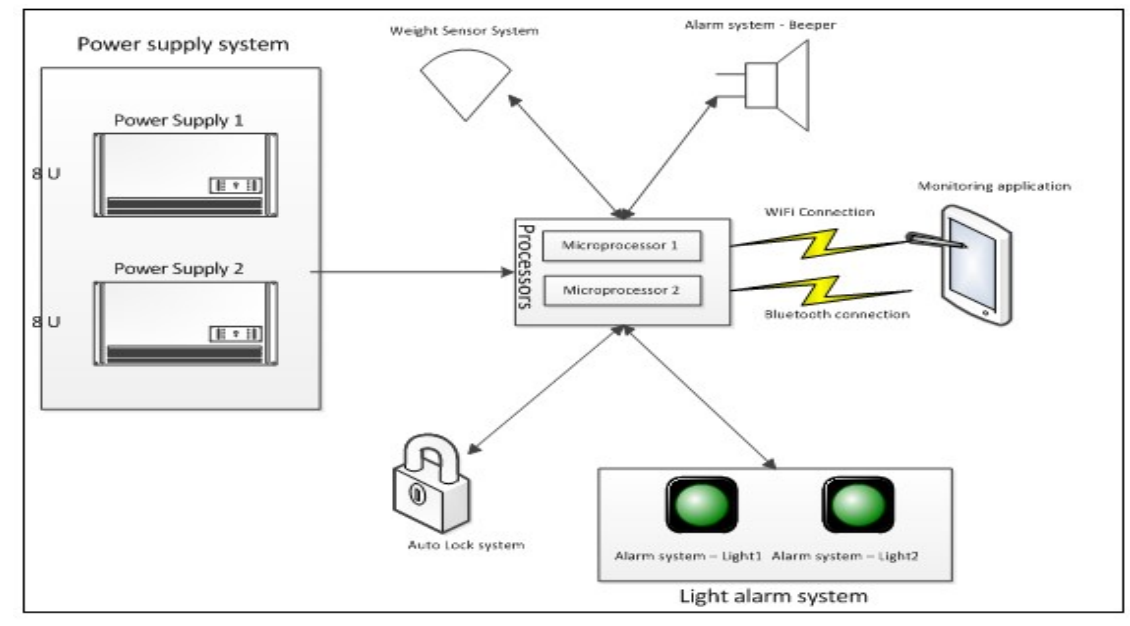

Fig. 3 Block diagram with implemented safety measures

Concerning the proposed safety-related system architecture, we have chosen to use redundent power supplies where the first one, the primary, is always selected. In case of failure, the secondary power supply will serve as a back up.

At processing control unit level, a safety-related 1002 architecture is targeted (one out of two).A 1002 architecture consists of two identical and idependant channels where the safety function is performed correctly if these channels are connected in such a way that one of them is sufficient for triggering the safety function [16]. One channel includes the processing control unit and its memory and peripherals. Furthermore, a safety-related weight sensor system is used. In this work, redundent sensor system is not used because of its size compared to the other components. Added 
to that, the use of diverse sensor that may detect the size and/or the shape of the medication could be an addition which would be analyzed in future works.

Based on the proposed concept, each processing unit is connected to the sensor system and will do its own calculations to check wheather the patient has taken the medication on time or not. A serial communication-based comparison, taken by the processing unit combined by additional diagnosis tests, will detect any failure behavior and trigger the safe state. In case of non-failure state, the processing units will unlock he medicine box, send alarm, determine the number of used pills,...

In case of failure state, the system will trigger the safe function which is defined in sending a message to the patient and to the ones using the phone application informing them that the system is not working properly and that they must handle and monitor the medication takeover manually. At the non-safe communication side, two different wireless communication configurations are established: a Wifi connection, which will be the primary transmission mode between the medicine box and the phone and a Bluetooth connection can be used as a backup in case of failure of the first solution. This will enhance the availability of the system.

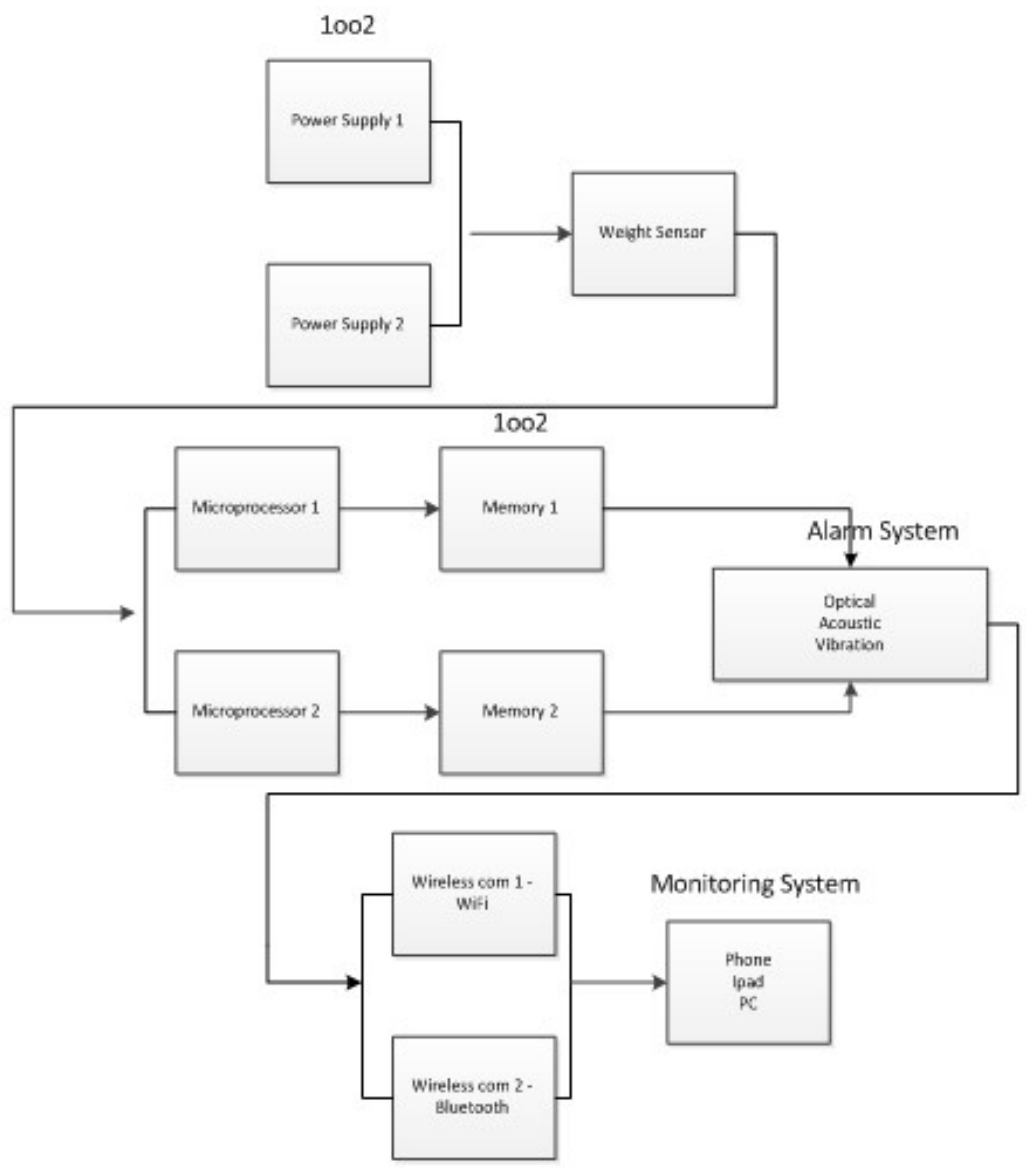

Fig. 4 Architectural design of the safe system 
International Journal of Biomedical Engineering and Science (IJBES), Vol. 5, No. 3/4, October 2018

\section{HARDWARE COMPONENTS}

This section will be dedicated to present the hardware components used to realize this system. Three main recommendations were taken into consideration when chosen these parts: the cost, the size and, most importantly, the accuracy.

\subsection{MiCROCONTROLLER}

Several controllers can be used to manage the instances of the proposed system. However, the Arduino Uno was chosen for its easy implementation, the several modules it enables as the Bluetooth connectivity that will be one of the connection possibilities between the hardware and the phone, its low price and its size[17].

Two main similar microcontrollers were used as presented in the safe system architecture shown in the previous section. The code embedded in these microcontrollers was presented in the flowchart of figure 2. The connections of each microcontroller are the weight sensor and the switch sensor, a light and a beeper alarms, a Bluetooth and WiFi connections, and the power supply.

Added to that, both microcontrollers can exchange data together. In fact, each one sends to the other a block of data informing it if the medication was taken correctly. Each processor compares its values to the second processor. Whenever one of the processors finds a difference between both outputs, the system enters the safe-state and a message is sent to the monitoring unit.

\subsection{SENSORS}

The main sensor used for the proposed application is the weight sensor. The accuracy of this sensor is of high importance as the pill may weight few milligrams. The measurement range may vary from few micrograms up to $500 \mathrm{~g}$. Several weight sensors are available and may be used. However, we have chosen to use the Arduino load cell weight sensor. The output of this sensor is analog and is converted to a digital representation using a 24 high precision $\mathrm{A} / \mathrm{D}$ converter chip HX711 [18].

The sensor measurement range is between $10 \mu \mathrm{g}$ and $500 \mathrm{~g}$ whereas the operational temperature ranges between $-5^{\circ} \mathrm{C}$ and $50^{\circ} \mathrm{C}$. Due to the high accuracy of the ADC module, the sensor circuitry is able to detect weight variation up to $3 \mu \mathrm{g}$.

However, the weight sensor is not the only sensor used in this system. The switch sensor is the second used sensor. For this latter, the lock sensor was deployed. It is normally closed. A $5 \mathrm{~V}$ sent by the microcontroller will enable to unlock it.

\subsection{CiRCUITRY}

After presenting the controller as well as the sensors and the types of connectivity with the mobile phone, this part will show the main circuits used to realize this system. The main importance of this part is to show how the duplications in the hardware were treated in order to reduce the errors to their lowest level.

Thus, figure 5 shows the whole circuit. It is clear that the weight sensor is connected to both micro controllers as well as the communication modules, the lock switch and the beeper. As for the LEDs, each microcontroller has its own elements. 


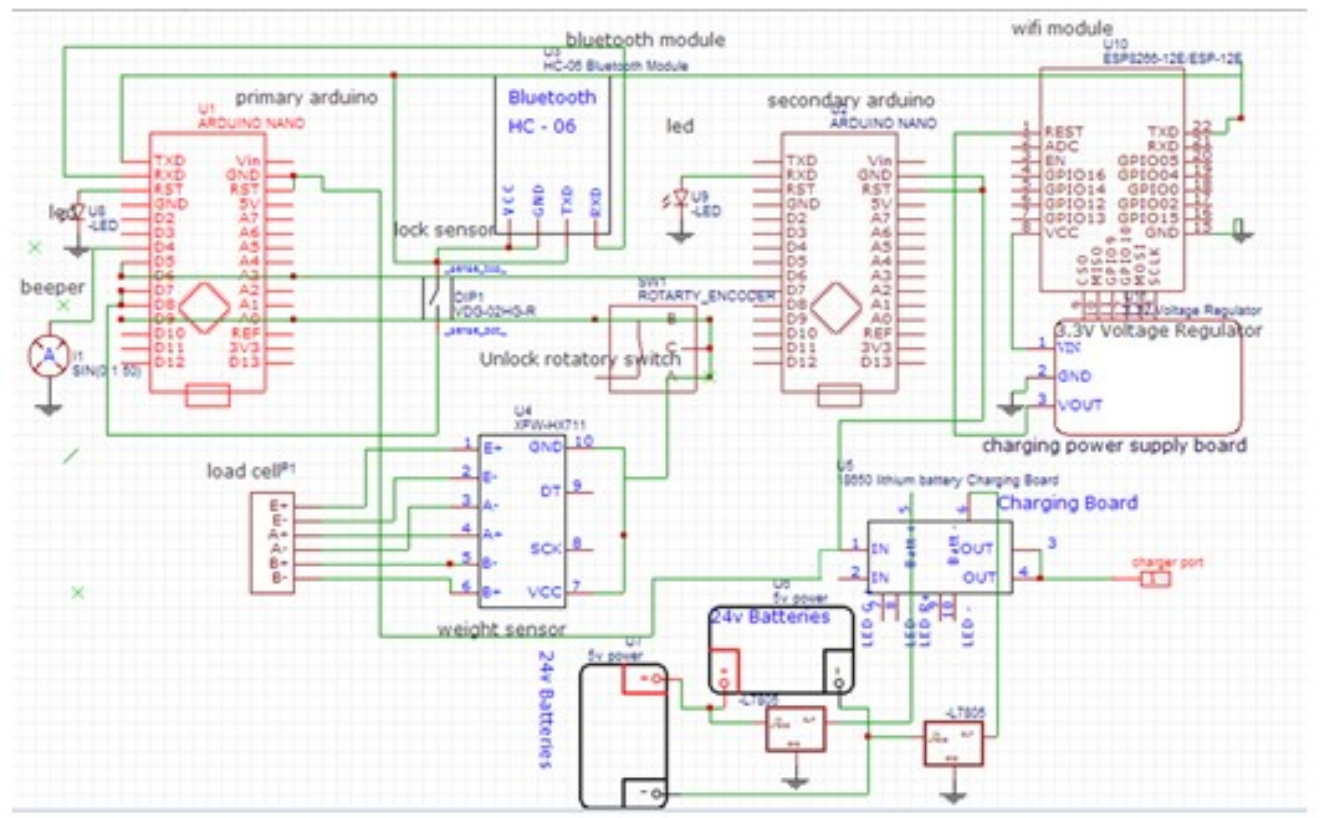

Fig. 5 - Electric circuits for the whole system

\subsection{SOFTWARE IMPLEMENTATION}

This section will present the main softwares developed in order to monitor, analyze and control the pills taken by the patient. Thus, it will be divided into two parts: the first part shows the microcontroller program (although it is mainly based on the flowchart shown in figure 2, there are still some important features concerning the safety issues that were not discussed in the first part of this paper) and the phone application program.

\subsubsection{Microcontroller Program}

Referring to the flowchart of figure 2, the main parts of the microcontroller program are included. However, this program lacks of two main parts, mainly for safety measurements:

- The measurement of the weight of the pills each time the medicine box is closed in order to define the number of remaining pills and to calculate the number of days/serving they may provide. Whenever the number of servings is below 5 or the number of days is below 3, an alarm is sent via the phone application to the patient and to his parents;

- The comparison of the results obtained by the primary arduino to those obtained by the secondary one and sent to the second processing unit. If both results are similar, the alarms are sent to the mobile application and the beeper is turned on (signals delivered by the primary arduino). As for the light, it is turned On/Off by each microcontroller.

Figure 6 shows a quick overview of the whole work done by each microcontroller. Note that, in order to limit duplications, the calculation of the pills taken as well as the check for the time of medication intake were not presented in this flowchart as they were explained in details in the flowchart of figure 2 . 


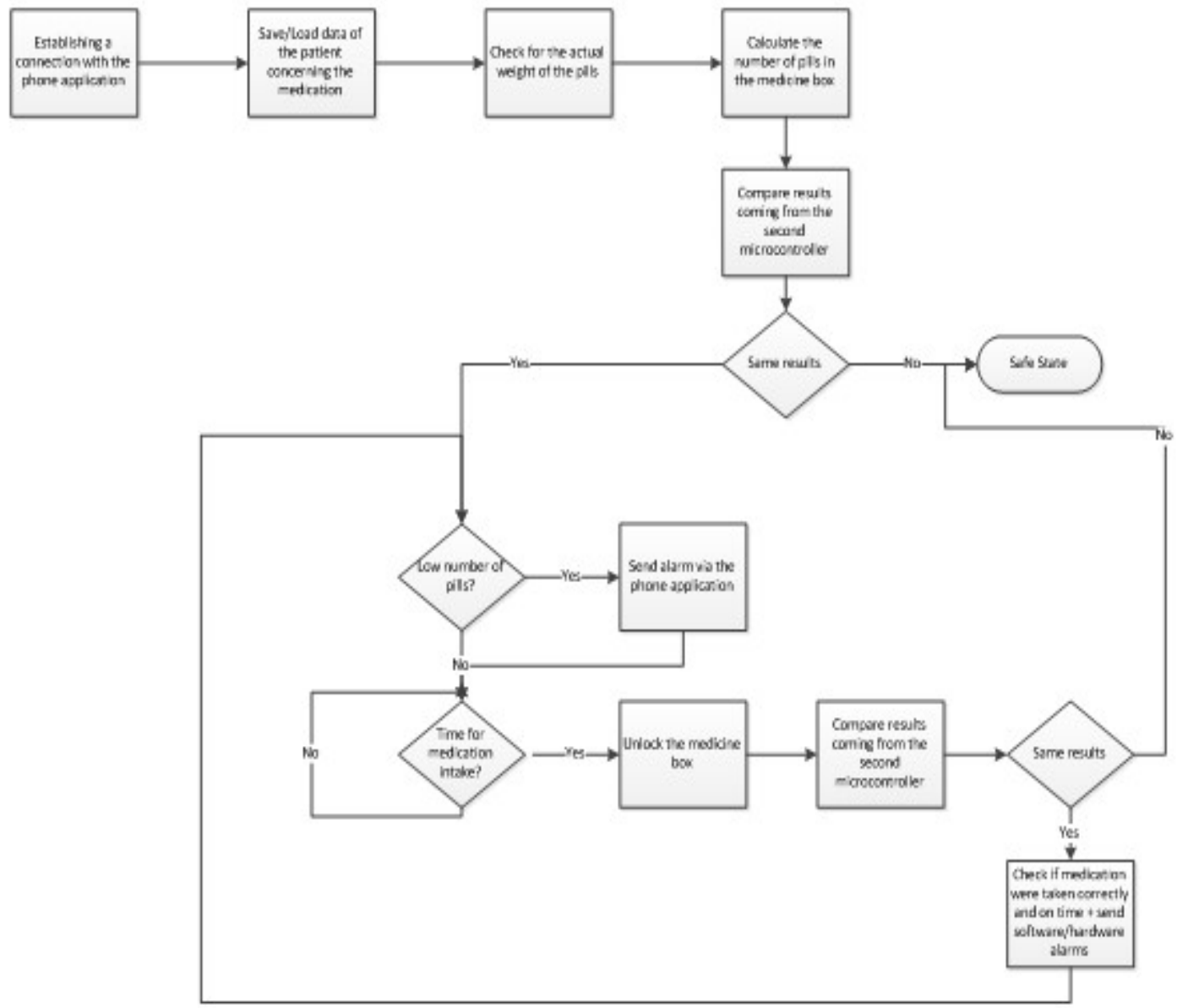

Fig. 6 Program of the processing units

\subsubsection{PHONE APPLiCATION}

The developed mobile application allows to save the name, age, prescription and the expiry date of the medicine pills and to send the dose and the time to the processor so that it will send back the number of pills and mention the unlock switch in the application.The android application was developed using MIT APP INVENTOR 2.

As for the procedure used, it is as follow:the user enters first one pill in the medicine box so that the weight can be recognized. Then, all piles are inserted in the medical box and the number of pills is displayed on the application. The user can, at any time, lock/unlock the medical box by sending the order to the primary microcontroller in order to close/open the door switch.

Figure 7 shows the layout of the application that the user will be using on his android mobile phone. 
International Journal of Biomedical Engineering and Science (IJBES), Vol. 5, No. 3/4, October 2018

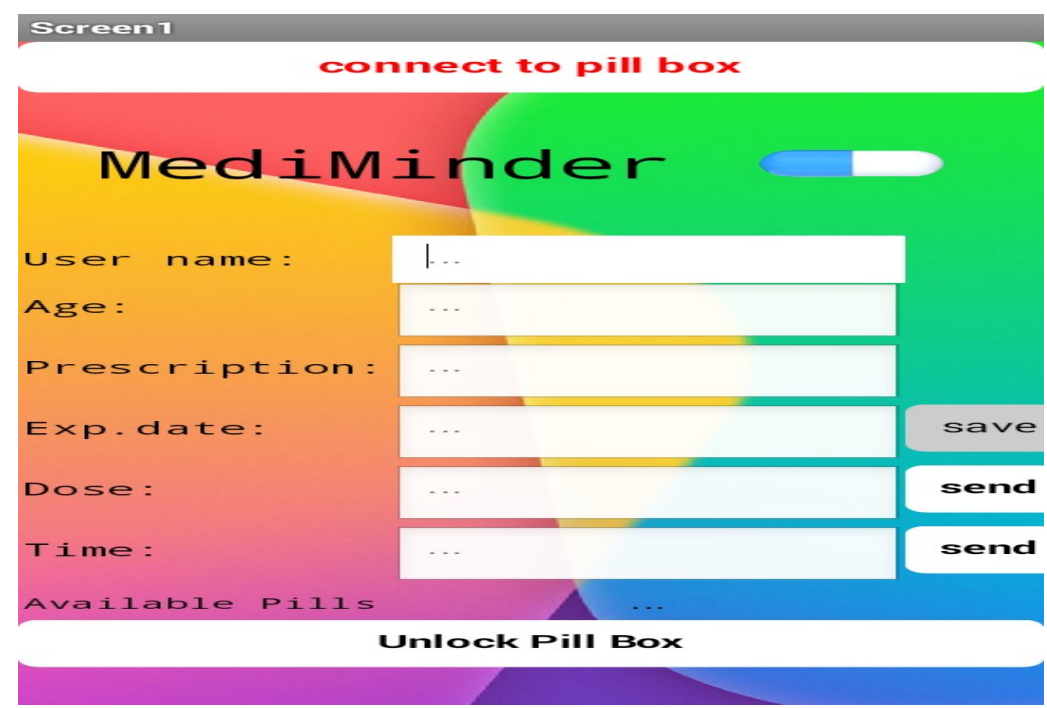

Fig. 7 Screenshot of the phone application

\section{Testing And VALidation}

Figure 8 shows a photo of the developed medicine box. Although its size remains of a major concern, the use of smaller weight sensor as well as the use of SMD electronic circuits would lead to a remarkable more compact system.

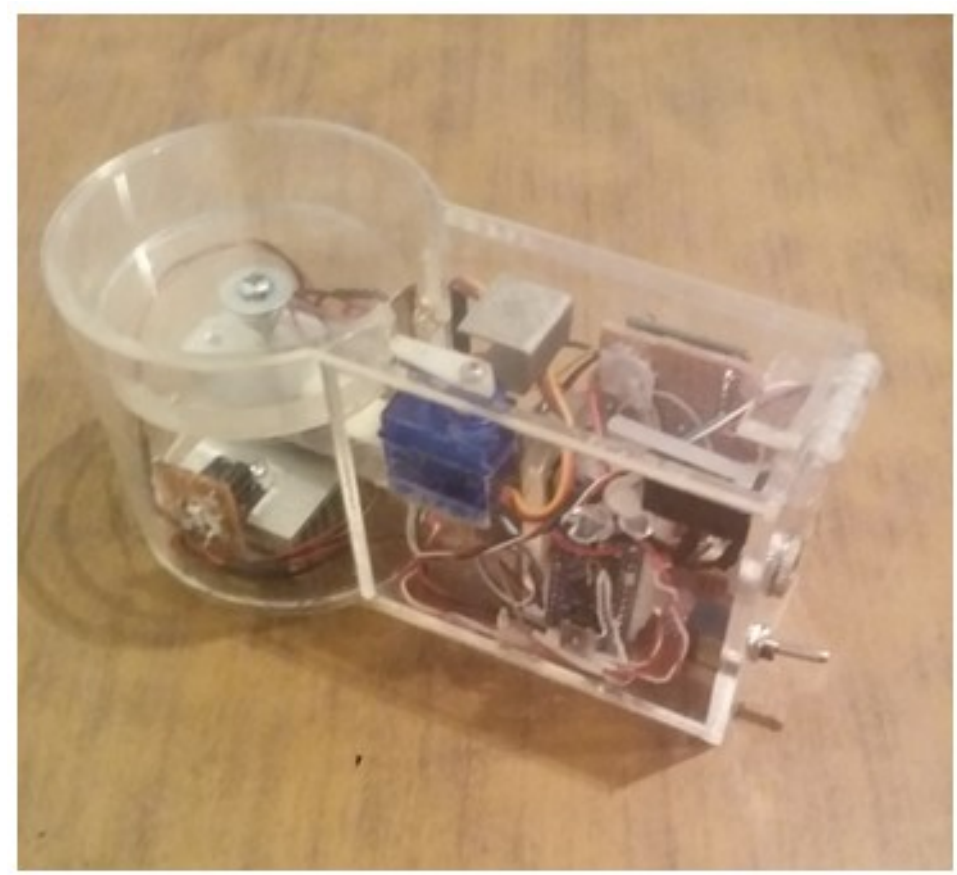

Fig. 8 - Photo of the developed medicine box

As for the testing, the proposed system was tested over around thirty patients considering different kinds of medicine pills of weight less than 500mg on different kinds of android phones 
with various dosage and time conditions, where the system can detect the correct dosage intake. Also the battery lifetime was tested where it gave successful results for about twelve hours without charging, and finally for the charging procedure the box produces a green light while charging which turns blue when it's full.

Figure 9 shows the results of a questionnaire distributed and filled by the patients having used this system.

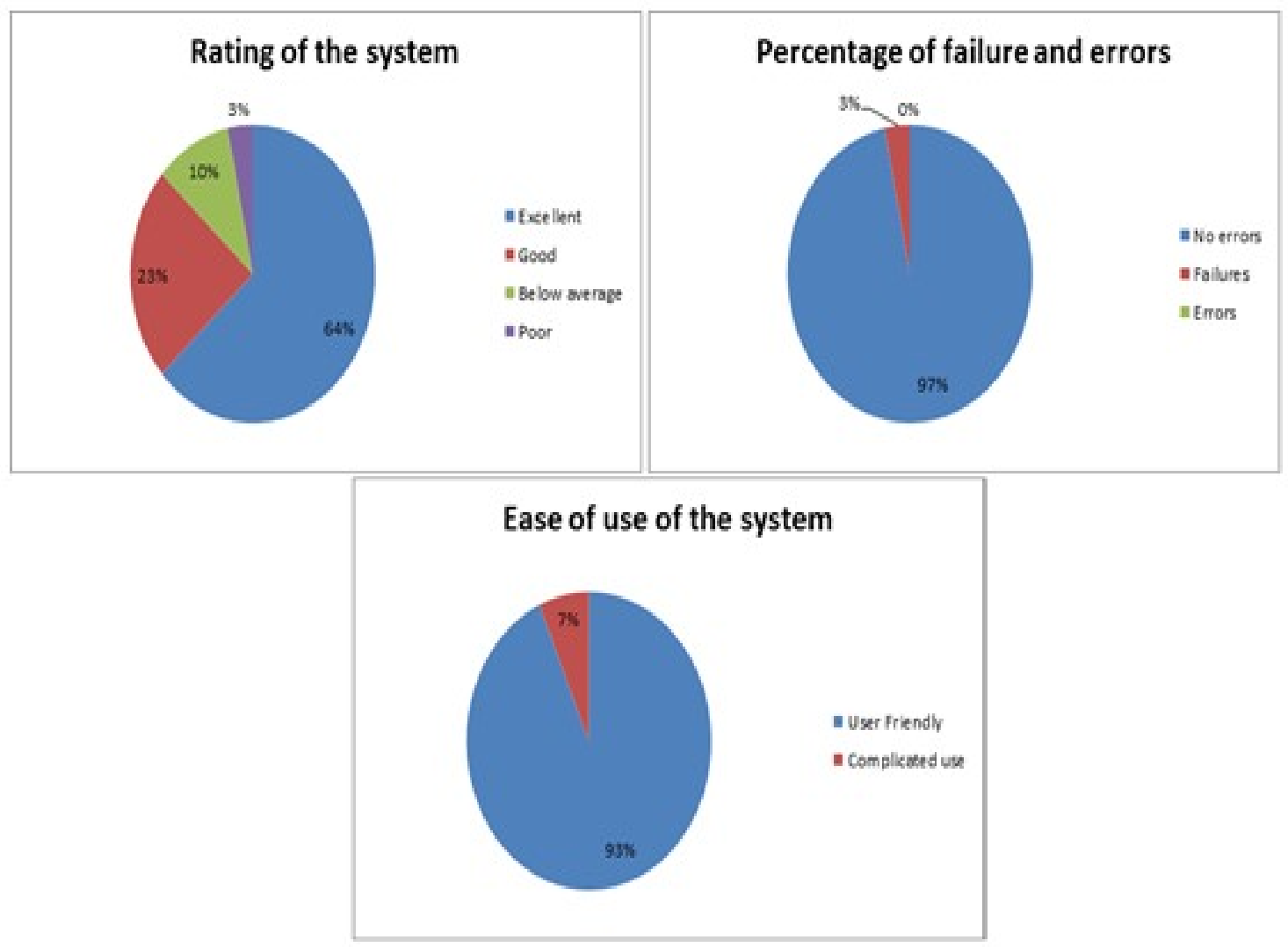

Fig. 9 Statistical info about the developed system

\section{Conclusion ANd Future Works}

The proposed project is, in its general purpose, aiming to control medication adherence. In this way, this smart pill box will help to increase life expectancy for those of older as well as younger aged adults. Additionally, the proposed device is low cost, user friendly, and above all, a safe system.

The suggested system is able to measure the weight of the pills, monitor the medicine intake, and remind the patient to take their medication during the allotted time. Furthermore, as a device that is connected to a smart phone, it does not disrupt the fast paced pattern of the patient's daily life, but rather accommodates to it. In addition, the 
device's use is suitable for all adults, including those with children. As a device that only unlocks through the application, it is safe to keep it anywhere as it is out of the reach of children. Indeed, this system was designed with a safety first approach in order to eliminate any additional risk to the patient.

As for some future works, we see this system as the beginning of a larger project for improving health care. The proposed device may be developed further through the below proposed measures:

- Adding more alarm systems and sensors as the ones listed in safety system section as the detection of the size of the pills to make sure of the taken medication;

- Compression of the size of the box to be compatible and more flexible for usage.

- Addition of more slides for the application to be used for multiple medications.

\section{REFERENCE}

[1] R. Abi Zeid Daou, T. Saadeh and R. Jahjah, "Controlling of Patient Environment Remotely by a Single Click," in Advances in Engineering Research, vol. 9, Nova, 2015.

[2] R. Ransing and M. Rajput, "Smart home for elderly care, based on Wireless Sensor Network," in International Conference on Nascent Technologies in the Engineering Field (ICNTE), Navi Mumbai, Indi, 2015.

[3] W. Ming, Y. Xiaoqing and F. Dina, "Design and implementation of home care system on wireless sensor network," in 8th International Conference on Computer Science \& Education (ICCSE), Colombo, Srilanka, 2013.

[4] N. Suryadevara and S. Mukhopadhyay, "Wireless sensors network based safe home to care elderly people: A realistic approach," in IEEE Recent Advances in Intelligent Computational Systems (RAICS), Trivandrum, India, 2011.

[5] Z. Li, W. Chen, J. Wang and J. Liu, "An automatic recognition system for patients with movement disorders based on wearable sensors," in IEEE 9th Conference on Industrial Electronics and Applications (ICIEA), Hangzhou, China, 2014.

[6] F. Fernández-Luque, J. Zapata and R. Ruiz, "A system for ubiquitous fall monitoring at home via a wireless sensor network," in IEEE Engineering in Medicine and Biology Society (EMBC), Buenos Aires, Argentina, 2010.

[7] C. Doukas and I. Maglogiannis, "Emergency Fall Incidents Detection in Assisted Living Environments Utilizing Motion, Sound, and Visual Perceptual Components," IEEE Transactions on Information Technology in Biomedicine, vol. 15, no. 2, pp. 277 - 289, March 2011.

[8] R. K. Megalingam, G. Pocklassery, V. Jayakrishnan, G. Mourya and A. A. Thulasi, "Smartphone based continuous monitoring system for home-bound elders and patients," in International Conference on Communications and Signal Processing (ICCSP), Melmaruvathur, India, 2014.

[9] B. Andò , S. Baglio, C. O. Lombardo and V. Marletta, "A multi-user assistive system for the user safety monitoring in care facilities," in IEEE International Workshop on Measurements \& Networking (M\&N), Coimbra, Portugal, 2015.

[10] International Electrotechnical Commission, "IEC 60601-1," 2005.

[11] K. Nikita, Safety Issues in Biomedical Telemetry, New York: Wiley-IEEE, 2014.

[12] S. Loznen, "Safety of bioinstruments and biomedical engineering education," in Nineteenth Convention of Electrical and Electronics Engineers in Israel, Jerusalem, Israel, 1996. 
International Journal of Biomedical Engineering and Science (IJBES), Vol. 5, No. 3/4, October 2018

[13] H.-L. Tsai, C. Tseng, L. Wang and F.-S. Juang, "Bidirectional smart pill box monitored through internet and receiving reminding message from remote relatives," in IEEE International Conference on Consumer Electronics - Taiwan (ICCE-TW), Taipei, Taiwan, 2017.

[14] A. Bharadwaj, D. Yarravarapu, S. Reddy, T. Prudhvi , K. Sandeep and O. Reddy, "Enhancing healthcare using m-care box (monitoring non-compliance of medication)," in International Conference on Innovative Mechanisms for Industry Applications (ICIMIA), Bangalore, India, 2017.

[15] S. Sohn, M. Bae, D.-K. Lee and H. Kim, "Alarm system for elder patients medication with IoTenabled pill bottle," in International Conference on Information and Communication Technology Convergence (ICTC), Jeju, South Korea, 2015.

[16] Y. Badamasi, "The working principle of an Arduino," in 11th International Conference on Electronics, Computer and Computation, Nigeria, 2014.

[17] K. Watkins, "Force, Load and Weight Sensors," in Sensor Technology Handbook, USA, Elsevier, 2005, p. 255-269. 